\title{
La aceptabilidad sensorial de los alimentos en puré ${ }^{1}$
}

\author{
Jamila R. Lepore y Wendy J. Dahl ${ }^{2}$
}

\section{Evaluación sensorial - ¿Qué es?}

La evaluación sensorial es el proceso en el que utilizamos nuestros sentidos (gusto, olfato, tacto, vista) y su aplicación para la determinación de la aceptabilidad de los alimentos. ¡Simplemente, se están evaluando los alimentos para asegurarse que se ven, huelen y saben delicioso!

\section{¿Por qué evaluar la aceptabilidad de los alimentos en puré?}

Es importante que disfrutemos los alimentos que comemos. Esto hace que tengamos una mejor calidad de vida. Esto no es diferente de aquellas personas que necesitan estar en dietas de purés debido a problemas de deglución $(1,2)$. El sabor y el aroma son aún más importantes con los purés debido a la modificación de la textura y de la apariencia.

Todos los purés deben ser degustados antes de ser servidos, particularmente las recetas nuevas. Al igual que usted no quiere servirle a la visita una receta sin antes probarlo, usted no quiere servir alimentos en puré sin saber si es apetecible.

\section{Evaluando los alimentos en puré}

Los alimentos en puré pueden ser degustados por el cocinero, el cliente, o ambos. Al probar las recetas nuevas, es una buena idea hacer que los que están consumiendo los alimentos los evalúen primero, así el cocinero puede hacer cambios en caso que sea necesario. Una manera fácil de hacer esto es el tener un grupo de degustación.
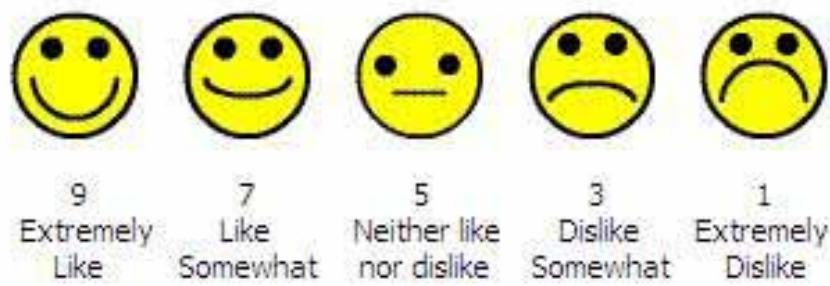

\section{5}

Neither like

nor dislike

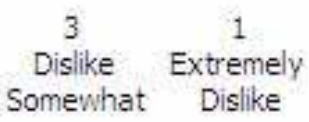

Figure 1. Las escalas de evaluación

Se presenta el nuevo puré y después hace que el grupo lo evalúe y proporcione su comentario crítico. Una escala de clasificación sencilla que puede ser usada es la escala de 9 puntos, en donde $1=$ me disgusta muchísimo, $5=$ no me gusta ni me disgusta, y el $9=$ me gusta muchísimo. Usando imágenes lo hace hasta más simple.

Preguntas que se deben usar para esta escala:

¿Cómo calificaría el sabor?

¿Cómo calificaría el aroma?

¿Cómo calificaría la apariencia?

¿Cómo calificaría la textura?

Las escalas de evaluación permiten comparar entre alimentos y entre individuos:

1. Entre alimentos - Por ejemplo, una receta de puré de brócoli puede obtener una calificación media de 5 mientras que un puré de zanahorias obtiene una calificación media

1. The English version of this document is FSHN12-13/FS206 Sensory Acceptability of Puréed Foods. Este documento, FSHN12-13s, es uno de una serie de publicaciones del Departamento de Food Science and Human Nutrition, Servicio de Extensión Cooperativa de la Florida, Instituto de Alimentos y Ciencias Agrícolas, Universidad de la Florida (UF/IFAS). Fecha de primera publicación diciembre de 2012. Visite el sitio web de EDIS en http://edis.ifas. ufl.edu/.

2. Jamila R. Lepore, RD, estudiante y Wendy J. Dahl, PhD, RD, profesora assistente, Departamento de Food Science and Human Nutrition, UF/IFAS, Gainesville, FL 32611. 
de 7. Esto sugiere que la receta de brócoli puede necesitar algún ajuste para mejorar su sabor. Alternativamente, es posible que las zanahorias sean simplemente más apetitosas que el brócoli. Usted tendrá que considerar qué calificación promedio será considerada inaceptable.

2. Entre individuos - Por ejemplo, un grupo de degustadores de sabores califican el puré de melocotones en una medida de 8 pero una persona lo califica como 1 . Usted puede querer determinar si a él/ella simplemente no le gustan los melocotones, o quizás no entendió la escala de calificación. A pesar de esa calificación baja, esa receta debe ser usada y es un alimento que se puede ofrecer ya que la mayoría del grupo la calificó en una escala alta.

Adjunto al final de este documento se puede encontrar una escala sensorial a la que le puede sacar copias para evaluar sus purés.

\section{Consejos para mejorar los alimentos en puré}

\section{El sabor}

El sabor de los alimentos en puré necesita ser un poco más intenso que los alimentos de textura regular. Esto se debe a que el acto de masticar libera sabores en la boca. Una vez que el alimento está en forma de puré, no requiere la masticación. Este puré simplemente se ingiere, sin que tenga mucho tiempo para que el sabor del alimento en puré sea degustado. Al aumentar el sabor de un alimento en forma de puré se ayuda al reconocimiento del sabor.

Hay varias maneras para lograr un sabor más fuerte y mejorado.

- Potenciadores de sabor son ingredientes para alimentos que pueden ser utilizados para aumentar el sabor natural de un alimento. Estos aumentan la intensidad del sabor. Estos ingredientes especiales son usualmente derivados de las proteínas o carbohidratos y pueden ser comprados. Las personas mayores han demostrado tener una disminución sensorial del gusto y del olfato $(3,4)$. Los potenciadores de sabor han demostrado mejorar la ingesta de alimentos en puré en las personas mayores de edad (5). Ejemplos de potenciadores de sabor (6):

$$
\begin{gathered}
\text { Glutamato de calcio } \\
5 \text { '-inosinato disódico } \\
\text { Etil Maltol } \\
\text { Glicina } \\
\text { L-ácido glutámico }
\end{gathered}
$$

\author{
L-Leucina \\ Glutamato de magnesio \\ Maltol \\ Glutamato monosódico (MSG) \\ Taumatina
}

- Cuando se ajusta la consistencia de los alimentos en puré, trate de usar alternativas líquidas de esos mismos alimentos en vez de agua. Por ejemplo, utilice caldo saborizado o jugos de las carnes, jugos de frutas para los purés de frutas y leche para productos lácteos y postres.

- Las especias y hierbas pueden ser también añadidas para aumentar la aceptabilidad de los alimentos, sin embargo éstas alteran el sabor natural de los alimentos y pueden hacer que sea más difícil identificar los alimentos en puré.

Hay cosas que se deben tomar en cuenta que pueden impactar negativamente el sabor (7). Estos incluyen la refrigeración y congelación, el calentamiento en microondas y el recalentamiento de los alimentos.

Estos métodos de almacenamiento y preparación pueden producir sabores desagradables, especialmente en alimentos que contengan grasas como muchas de las carnes, mariscos y productos lácteos (7). Si bien estos procesos son necesarios en la producción de alimentos, hay algunas cosas que se pueden hacer para maximizar los sabores agradables y reducir al mínimo los sabores desagradables.

- Siempre que sea posible, trate de preparar los purés con los ingredientes más frescos y sirva inmediatamente. Si necesita preparar los alimentos con uno o dos días de anticipación, es mejor congelar los alimentos que refrigerarlos.

- Cuando la refrigeración y el almacenamiento son inevitables, limite el tiempo en que lo alimentos pasan en el almacenamiento. Esto es especialmente cierto para los alimentos que ya han sido preparados, como los purés. Mantenga el almacenamiento de los purés hechos en casa el tiempo más corto posible. Los purés preparados comercialmente están diseñados para ser congelados y almacenados por mucho más tiempo.

- Puede que no sea práctico preparar y servir purés inmediatamente, por lo que el recalentamiento es inevitable. Cuando se recalientan los alimentos, es mejor prepararlos usando métodos convencionales como la estufa o el horno ya que producirá un puré de mejor calidad. 
- Evite usar el horno microondas para asegurar la mejor calidad en sus purés. El calendar y/o recalentar en el horno microondas puede causar sabores desagradables, especialmente con carnes y mariscos. Restringir el uso del horno microondas a productos que son específicamente diseñados para ser calentados en el horno microondas, como los vegetales congelados o platos que tienen instrucciones de uso en el horno microondas.

Es muy importante no combinar alimentos en puré ya que el sabor puede llegar a ser irreconocible. La inhabilidad de identificar un alimento (por ejemplo, ¿Es esto pollo o carne de cerdo?) ha sido asociado con una experiencia negativa por aquellos en dietas de purés (8). Por ejemplo, es mejor presentar los purés como elementos individuales o como capas en vez de mezclarlos en un plato de tipo "Cacerola". Por ejemplo, pasta en puré, puré de albóndigas de carne, puré de tomates pueden ser colocados en capas para que cada sabor sea reconocido. Es importante recordar que cuando la textura se modifica, la dependencia al sabor para el reconocimiento de los alimentos es mayor.

\section{El aroma}

El aroma es uno de los primeros sentidos que usamos durante las comidas. A menudo es lo primero que notamos, incluso antes de que el plato llegue a la mesa. ¡Por esto es que es tan importante asegurarse que los alimentos en puré huelan bien! Al igual que con el gusto, lo mejor es limitar el número de alimentos combinados en el puré, para que el aroma de este pueda ser reconocido. Piensa en ello. Si siente un olor a jamón, pero come un puré hecho de jamón, papas y judías verdes, probablemente puede que nos sepa desagradable porque no es lo que esperábamos. Esto se debe a que el aroma es inherentemente vinculado al gusto.

Cuando un alimento se calienta, el aroma se libera. Los aromas son mejormente preservados cuando los alimentos son calentados por medios convencionales (estufa, horno). Aunque el horno microondas es adecuado para el recalentamiento de alimentos en puré, el aroma puede ser alterado. Como el calor del horno microondas es desigual en el alimento, el aroma se libera en diferentes tiempos y diferentes lugares del alimento. Como resultado, algunos aromas se pierden en el horno microondas, mientras otros se mantienen en el alimento. Esto altera el perfil general de olor del alimento cuando se consume (9).

Es también posible alterar el aroma de un alimento en puré al añadir ingredientes que producen aromas potentes. Un alimento que se hace en forma de puré, se le puede mejorar su aroma al añadirle hierbas, especias y/o extractos (por ejemplo, vainilla). Los potenciadores de aromas comerciales están disponibles, el más común es el glutamato monosódico (MSG) y 5'-monofosfato de inosina (5'IMP) (10).

\section{La textura}

¡Una textura aceptable es una de las características más importantes de los alimentos en puré! Hay varias cosas que se deben tener en cuenta al preparar un alimento en puré para obtener una consistencia adecuada.

Asegúrese que los alimentos en puré sean suaves y cohesionados. Un puré no debe requerir masticación y debe ser "espeso en la cuchara." No debe ser ni muy seco ni muy delgado. Los purés no deben contener grumos.

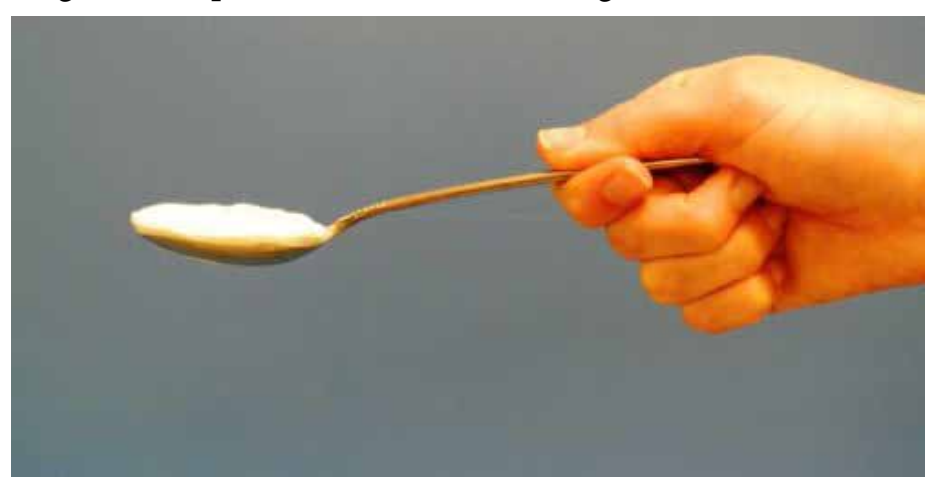

Figure 2. El puré debe sostenerse en la cuchara como el yogurt/pudín.

Los alimentos en puré no pueden ser pegajosos. Por ejemplo, el puré de papas y otros alimentos almidonados pueden ser espesos y adherirse al paladar. El añadir crema agria o una salsa puede reducir la pegajosidad y producir una textura más suave y cremosa. Para disminuir la pegajosidad de los alimentos con almidón en puré, utilice salsas, cremas u otras grasas adecuadas para el plato que se está preparando.

Los purés no deben ser demasiado delgados. Los purés delgados generalmente son más difíciles de digerir que los purés “espesos en la cuchara”. Un puré muy delgado es normalmente el resultado de añadir mucho líquido durante su preparación. Para lograr la textura de "espesor de cuchara”, añada líquido un poco a la vez.

En la preparación de alimentos en puré, es posible que haya la necesidad de usar un espesante. Si bien hay muchos espesantes de almidón comercial disponibles (por ejemplo, Thick-It o Thickenup $^{\oplus}$ ), el utilizar alimentos para espesar los purés hace que el producto sea generalmente más nutritivo y apetitoso. Los alimentos del grupo de granos funcionan bien para espesar purés. Algunos ejemplos de estos incluyen: 
Galletas en polvo/Migas de pan

Cereales para bebés

Cereales finamente molidos para el desayuno

Puré de garbanzos o judías blancas

Puré de banana

Puré de vegetales con almidón (papas, guisantes)

\section{La apariencia}

La apariencia es una característica tan importante como lo son las otras características en los alimentos. A menudo "Comemos con nuestros ojos" primero. El color se vuelve más importante con los alimentos en puré ya que la apariencia es significativamente alterada.

Se ha demostrado que los adultos mayores dependen más de las señales visuales para identificar los productos alimenticios (11). El reconocimiento de los alimentos es una parte importante en la experiencia de la comida para los mayores que están en dietas de alimentos en puré (8). Debido a que un alimento en puré ya no tiene su forma original, hay algunas maneras de hacerlo más reconocible y atractivo.

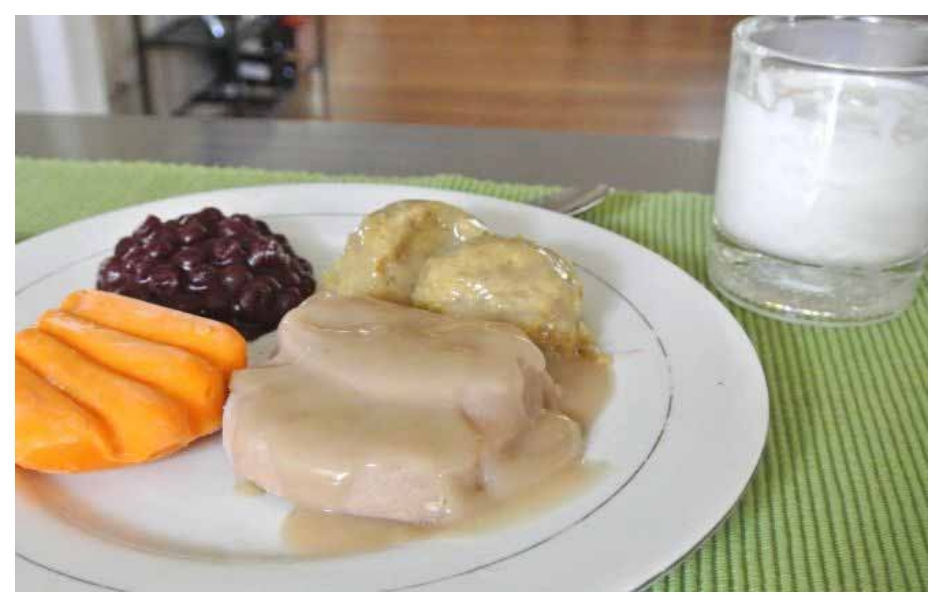

Figure 3. Sirva la comida en puré en platos atractivos

- Mantenga los alimentos separados tanto como sea posible. Por ejemplo, la combinación de puré de zanahorias con puré de guisantes producirá un color poco atractivo. Al mantener los alimentos separados, el color se mantiene y se hace más identificable.

- Considere el uso de moldes para alimentos en puré. Estos moldes están diseñados para hacer el puré lo más parecido al alimento original completo. El uso de moldes puede o puede no ser el preferido. Los moldes de alimentos pueden mejorar la apariencia y mejorar el consumo para algunos individuos (12). Para otros, es preferible un puré no moldeado. Es posible que el puré moldeado, que tiene la forma original del alimento, cree la expectativa que el alimento tendrá la misma sensación en la boca como el alimento original. Asegúrese de preguntar si los purés moldeados son los preferidos.

- El añadir guarniciones al puré también puede mejorar la apariencia. Esto es especialmente cierto si usted esta usando cucharas en vez de moldes de alimentos. Las guarniciones para los purés incluyen los siguientes:

Salsas

Condimentos (salsa de tomate, mostaza, mayonesa, etc.) Mantequilla/Margarina

Crema agria

Crema batida

Jarabes gruesos

\section{Resumen y vínculos útiles}

$\mathrm{Al}$ intentar nuevas recetas o usar recetas existentes para purés, asegúrese que este llame la atención a todos los sentidos. El puré debe oler, saber y verse muy apetitoso. Es muy importante que la textura sea suave y cohesiva. ¡La mejor manera de evaluar si su receta atrae todas estas cualidades, es hacer pruebas sensoriales! Esto puede ser realizado por el chef, cuidador y/o la persona(s) con disfagia. Por favor vea el formulario adjunto y utilícelo para evaluar sus purés.

A continuación se enumeran las publicaciones útiles para su referencia:

Comidas en puré para problemas al tragar (1)

Problemas para tragar y el adulto mayor (2)

\section{Referencias}

(1) Dahl WJ. Puréed Foods for Swallowing Problems. 2011

[cited; Available from: http://edis.ifas.ufl.edu/pdffiles/FS/ FS16800.pdf

(2) Dahl WJ. Swallowing Problems and the Older Adult. 2011 [cited; Available from: http://edis.ifas.ufl.edu/pdffiles/ FS/FS16400.pdf

(3) Schiffman SS, Warwick ZS. Use of flavor-amplified foods to improve nutritional-status in elderly persons. Annals of the New York Academy of Sciences. 1989;561:267-76.

(4) Schiffman SS, Warwick ZS. Effect of flavor enhancement of foods for the elderly on nutritional status, food intake, biochemical indexes, and anthropometric measures. Physiology \& Behavior. 1993;53:395-402. 
(5) Mathey M-F, Siebelink E, de Graaf C, Van Staveren WA. Flavor enhancement of foods improves dietary intake and nutritional status of elderly nursing home residents. Journal of Gerontology. 2001;56A:M200-5.

(6) Administration FaD. Food Additives: Alphabetical List. 2002.

(7) Flavor, Fragrance, and Odor Analysis. New York: Marcel Dekker, Inc.; 2002.

(8) Blaise M. Mealtime experiences of hospitalized older patients requiring a puree consistency diet: University of Montreal; 2009.

(9) Roberts DD, Pollien P. Analysis of Aroma Release during Microwave Heating. Journal of Agriculture \& Food Chemistry. 1997;45:4388-92.

(10) Astray G, García-Ríob L, Mejuto J, Pastrana L. Chemistry in Food: Flavours. Electronic Journal of Environmental, Agricultural, and Food Chemistry. 2007;6:1742-63.

(11) Philipsen DH, Clydesdale FM, Griffin RW, Stern P. Consumer age affects response to sensory characteristics of a cherry flavored beverage. Journal of Food Science. 1995;60:364-8.

(12) Cassens D, Johnson E, Keelan S. Enhancing taste, texture, appearance, and presentation of pureed food improved resident quality of life and weight status. Nutrition Reviews. 1996;54:S51-4. 
Copia de archivo: para las recomendaciones actuales, consulte http://edis.ifas.ufl.edu o su oficina de extensión local.

Forma para comentarios.

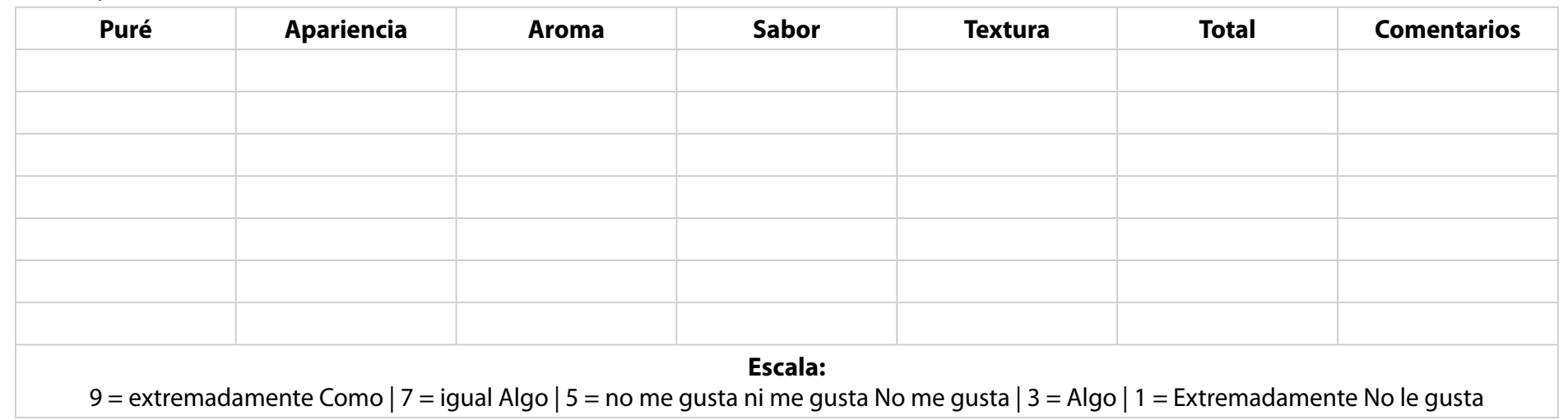

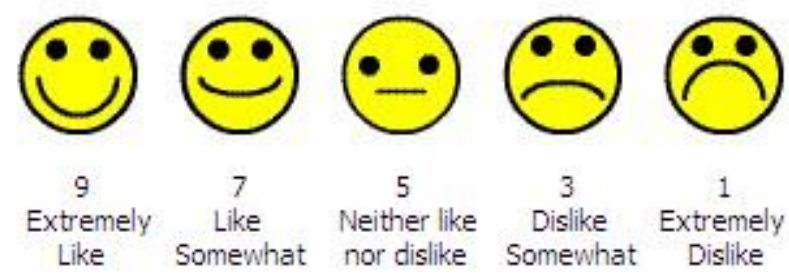

